\title{
Caracterização da clientela atendida no Núcleo de Psicologia Aplicada da Universidade Federal do Espírito Santo
}

\author{
Rita de Cássia Ramos Louzada \\ Universidade Federal do Espírito Santo
}

\begin{abstract}
Resumo
Este artigo apresenta o perfil da clientela do Núcleo de Psicologia Aplicada da Universidade Federal do Espírito Santo, de acordo com as seguintes variáveis: sexo, idade, renda familiar, escolaridade, local de moradia, ocupação, motivo da consulta e forma de encaminhamento. Com base nos dados encontrados são feitas reflexões em torno da estruturação deste tipo de serviço, suas articulações com os serviços de saúde mental da rede pública e a formação de psicólogos no âmbito do Estado do Espírito Santo.
\end{abstract}

Palavras-chave: saúde mental; perfil de clientela; saúde pública

\begin{abstract}
Profile of the clients assisted at "Núcleo de Psicologia Aplicada" (Applied Psychology Center) of the Federal University of Espírito Santo. This article presents the profile of the clients assisted at "Núcleo de Psicologia Aplicada” of Federal University of Espírito Santo, according to the following variables: sex, age, family income, education, place of residence, occupation, reasons for consultation and types of assistance provided. Based on data obtained, some reflections are presented in regard to the organization of this kind of service, its relations with public mental health services and the professional preparation of psychologists in the state of Espírito Santo.
\end{abstract}

Keywords: mental health; client profile; public health

A Organização Mundial de Saúde (OMS) preconiza que, para melhorar a saúde mental, há que se investir na produção de dados concretos sobre os serviços e recursos existentes e definir uma estratégia de avaliação contínua de toda nova atividade (Saraceno, Asioli, \& Gianni, 1994). Segundo Saraceno et al. (1994), para se alcançar o aperfeiçoamento dos serviços de saúde mental, tal como apontado pela OMS, se faz necessária uma “atitude epidemiológica”, que possibilite ratificar que o manejo do sofrimento psíquico não se limita a um aplicar meticuloso de técnicas, e sim, deve ser considerado como invenção permanente.

No campo da saúde mental, e no que tange à realidade brasileira, a literatura aponta alguns trabalhos envolvendo a identificação da população que busca atendimento nas diversas instituições e serviços. Urtiaga, Almeida, Vianna, Santos e Botelho (1997) estudaram os fatores preditivos de abandono em psicoterapia numa clínica, na cidade de Pelotas-RS. Embora não visassem definir o perfil da clientela, oferecem-nos dados a esse respeito: a maior parte dos clientes era do sexo feminino
(77\%), com idade entre 20 e 39 anos (70,1\%), solteiros (63,2\%), estudantes (35,1\%) e com renda de até 4 salários-mínimos (65,2\%). A busca por atendimento foi predominantemente espontânea e as queixas relacionavam-se mais a dificuldades no relacionamento em geral e com a família (42,6\%).

Outra pesquisa recente de grande envergadura foi a realização do I Censo da População de Internos nos Hospitais Psiquiátricos da Cidade do Rio de Janeiro. Os dados quantitativos a respeito do Censo nos são oferecidos por Fagundes e Libério (1997): 66\% da clientela era do sexo masculino, 70\% estava em idade produtiva, 47,3\% não dispunha de qualquer vínculo previdenciário e 37,2\% tinha renda própria. Foram encontrados 12,7\% em primeira internação e a maior parte dos usuários com altas taxas de reinternações. Em 56,9\% da clientela o tratamento era a internação (e apenas isso) e 61,9\% dos internos não recebiam outra modalidade de atendimento senão a consulta psiquiátrica. Os autores indicam que o desafio hoje se coloca numa maior qualificação da assistência articulada com o desenvolvimento de políticas de reabilitação psicossocial. 
No âmbito da Universidade Federal do Rio de Janeiro, Campos e Fortes (1992, citado por Brasil, 1998) identificaram o perfil da clientela atendida no Ambulatório do Serviço de Psicologia Médica e Saúde Mental do Hospital Universitário Clementino Fraga Filho, durante o ano de 1989. As características levantadas apontavam para pacientes predominantemente do sexo feminino (69\%), que residiam na área programática do ambulatório (57\%) e encontravam-se, em sua maioria, em idade economicamente ativa.

Especificamente quanto às clínicas-escola, foi possível verificar que a maior parte das pesquisas produzidas em nosso meio articulam os perfis de clientela aos diversos serviços oferecidos pelas instituições, com importantes questionamentos sobre a adequação das práticas existentes.

Lopez (1983) estudou, na cidade de São Paulo, o perfil de 2.826 pacientes atendidos em clínicas-escola de Psicologia, durante o ano de 1977. Nessa época, a população das clínicas constituía-se prioritariamente de crianças em idade escolar (6 a 15 anos) e de mulheres adultas (16 a 50 anos). Destaca-se a “quase ausência” de homens no grupo estudado e de um pequeno grupo de mulheres idosas. A maior parte dos clientes compareciam espontaneamente e apresentavam distúrbios de ordem afetiva. Na discussão, a autora afirma que os serviços estudados foram criados para atender a população, “... porém grande parte da clientela não é chamada ou é encaminhada para fora (...) e em pouquíssimos casos as clínicas podem dizer ter realizado um trabalho completo.” (p. 130).

Essa situação é remetida aos conflitos existentes no campo técnico e teórico, na identidade e na função social dos profissionais de Psicologia. Conclui a autora pela necessidade de redefinição da função do psicólogo, considerando-se as condições sociais em que se insere.

Outro estudo, também realizado no estado de São Paulo, abordou a clientela da clínica-escola do Instituto de Psicologia da PUCCAMP (Carvalho \& Térzis, 1989). Os dados foram coletados a partir de 2.102 prontuários de pacientes. Os resultados mostraram que - de 1970 até 1985 - foram atendidas, na clínica, pessoas de ambos os sexos, predominantemente na faixa etária de 6-10 anos, solteiros, estudantes e com escolaridade de nível primário. Os pacientes, na maioria, eram encaminhados por instituições escolares. Em suas conclusões, os autores afirmam que os períodos de transição no desenvolvimento individual (início da escolarização, puberdade, gravidez, casamento, etc.) "têm grande probabilidade de estarem associados a uma maior incidência de crises” (p. 108). Consideram ainda que este tipo de estudo pode auxiliar tanto na melhoria do atendimento à comunidade quanto na formação dos alunos de cursos de Psicologia.

Santos (1990), por sua vez, examinou a clientela de uma clínica psicológica voltada para atendimento infantil, localizada na cidade de São Paulo. Dentre o universo de pacientes atendidos durante o ano de 1985 (182 pacientes) foi sorteada uma amostra de 65 casos. Suas conclusões apontam para uma homogeneização quanto ao nível profissional e educacional dos responsáveis pelos clientes, constituído em sua maioria, por pessoas de baixo nível sócio-econômico. A clientela encontrada era originária de famílias de trabalhadores manuais com pouca ou nenhuma qualificação, com renda menor que o salário mínimo per capita. Santos conclui que fora do âmbito institucional público, seria bastante improvável que essa população tivesse qualquer tipo de acompanhamento psicológico. Nesse mesmo texto, no que se refere à revisão bibliográfica realizada, é possível perceber a existência de vários estudos sobre a prática clínica, especialmente de psicólogos. Esses estudos tentam, na maioria, mapear serviços de atendimento psicológico comunitário, caracterizando clientela, conceituando o trabalho psicológico em instituições, propostas alternativas para atendimento em nível institucional, entre outros. Embora Santos (1990) tenha encontrado trabalhos desse gênero no Brasil desde 1959, a maior parte deles foi produzida ao longo da década de 80. Segundo sua avaliação, ainda há muito o que produzir nessa área. E, embora pouco numerosos, admite que estudos dessa natureza fornecem um acervo apreciável para o desenvolvimento de novas hipóteses de pesquisa e aperfeiçoamento metodológico.

Santos, Moura, Pasian e Ribeiro (1993) centraram-se na clientela adolescente de uma clínica-escola da Faculdade de Filosofia Ciências e Letras da Universidade de São Paulo, em Ribeirão Preto. Foram consultados 208 prontuários, no período de 1987 a 1989. Os resultados mostraram que a clientela constituía-se de indivíduos do sexo feminino, solteiros, católicos, com idade entre 13 e 32 anos, escolaridade secundária ou universitária, com residência em Ribeirão Preto e arredores. Alguns pacientes exerciam atividades profissionais específicas - em sua maioria de nível inferior, ou de baixo nível técnico - ou apenas estudavam. Os autores tecem considerações a respeito da precária definição da identidade do profissional de Psicologia, afirmando que as clínicas-escola e as instituições formadoras vêm ratificando a distância entre os serviços oferecidos e as reais necessidades da população.

Reconhecendo a importância dos dados levantados e das discussões empreendidas nos trabalhos anteriores, delineou-se a presente pesquisa, com o objetivo de traçar o perfil da clientela do Núcleo de Psicologia Aplicada (NPA) da Universidade Federal do Espírito Santo (UFES), durante o ano de 1996.

\section{Método}

Trata-se de pesquisa documental, com dados coletados a partir das fichas dos pacientes atendidos no NPA/UFES. Dentre a população recebida durante o ano de 1996 (219 pacientes), foi sorteada uma amostra de 90 fichas, utilizandose uma tabela de números equiprováveis.

A caracterização da clientela foi feita a partir das seguintes variáveis: local de moradia, sexo, idade, escolaridade, profissão, nível sócio-econômico (expresso através da renda familiar), motivo da consulta (queixa), forma de encaminhamento à instituição. Para a variável profissão utilizou-se uma categorização adaptada a partir de Urtiaga et 
al. (1997), incluindo-se o trabalho doméstico e a situação de aposentadoria.

Embora existissem no NPA uma grande variedade de projetos de estágio, que incluíam atividades fora de seu espaço físico, junto a determinadas comunidades ou outras instituições, considerou-se nesta pesquisa apenas os clientes que passaram pelo "plantão de acolhimento", no qual todos os pacientes de primeira vez eram entrevistados. Ou seja, considerou-se apenas as fichas dos sujeitos que chegaram ao NPA demandando algum tipo de tratamento psicológico.

Os resultados encontrados foram tabulados e inseridos nas informações gerais sobre o NPA, colhidas a partir de entrevista com a coordenadora do núcleo.

\section{Resultados e Discussão}

O Núcleo de Psicologia Aplicada (NPA) da Universidade Federal do Espírito Santo (UFES), está localizado no bairro de Goiabeiras, em Vitória, capital do estado. Segundo informações colhidas junto à sua coordenação, o NPA não é um órgão autônomo, vincula-se ao Curso de Graduação em Psicologia, não possuindo qualquer relação formal com o Sistema Único de Saúde (SUS). À época da pesquisa, atendia gratuitamente pessoas da comunidade que buscavam espontaneamente a instituição ou chegavam encaminhadas formalmente. A inscrição para atendimento ocorria em período específico e a recepção era feita, através do "plantão de acolhimento", por um aluno-estagiário, que levantava as informações básicas sobre o paciente e o motivo da consulta. Apoiando-se nesses dados, os estagiários encaminhavam, sob supervisão, os pacientes aos diversos projetos de estágios existentes no NPA.

A maior parte da clientela identificada (50\%) residia em Vitória (ver Tabela 1) e, em segundo lugar, observou-se que $27,8 \%$ dos pacientes eram oriundos do município da Serra.

Tabela 1

Distribuição de freqüência conforme o local de moradia

\begin{tabular}{lcc}
\multicolumn{1}{c}{ Local de moradia } & $\mathrm{f}$ & $\%$ \\
\hline Vitória & 45 & 50 \\
Serra & 25 & 27,8 \\
Cariacica & 11 & 12,2 \\
Vila Velha & 9 & 10 \\
\hline Total & 90 & 100 \\
\hline
\end{tabular}

Nota: considerou se em todas as tabelas apenas uma casa decimal no cálculo da porcentagem.
Isto faz pensar não só na proximidade de determinadas cidades em relação à capital, onde se localiza o NPA (Vitória), como também na situação da rede de serviços de saúde mental existentes nos outros municípios referidos. Isto se justifica porque em algumas fichas aparecia a referência à procura do NPA em função da gratuidade, além da dificuldade de vagas nos postos da rede pública de assistência.

Quanto ao sexo observou-se quase uma equivalência na freqüência: 48,8 \% de homens e 51,1\% de mulheres, como se observa na Tabela 2 .

Tabela 2

Distribuição de freqüência conforme o sexo

\begin{tabular}{lcc}
\hline \multicolumn{1}{r}{ Sexo } & $\mathrm{f}$ & $\%$ \\
\hline Masculino & 44 & 48,8 \\
Feminino & 46 & 51,1 \\
\hline Total & 90 & 100 \\
\hline
\end{tabular}

Com relação à faixa etária (Tabela 3), pôde-se perceber maior concentração de clientes entre 20 a 29 anos (28,8\%). Vale ainda totalizar as freqüências das três faixas etárias iniciais, obtendo-se o percentual de 79,8\% entre 0 a 29 anos, caracterizando uma procura maior por atendimento a crianças, adolescentes e adultos jovens.

Tabela 3

Distribuição de freqüência conforme a faixa etária

\begin{tabular}{lcc}
\hline Idade & f & $\%$ \\
\hline $\mathbf{0}-\mathbf{9}$ & $\mathbf{2 4}$ & 26,7 \\
$10-19$ & 22 & 24,4 \\
$20-29$ & 26 & 28,9 \\
$30-39$ & 16 & 17,8 \\
$>40$ & 2 & 2,2 \\
\hline Total & 90 & 100 \\
\hline
\end{tabular}

A renda familiar (Tabela 4), apresentou variação de 0 a 23 salários mínimos. Evidentemente, este último foi o valor máximo encontrado e não era regra. Cerca de 55,4\% dos pacientes atendidos ocupavam a faixa de 0 a 6 salários-mínimos ${ }^{1}$. Porém, há que se considerar a chegada de pessoas que possuem renda mais alta e os motivos que as levam a procurar um serviço de atendimento público. 
Tabela 4

Distribuição de freqüência conforme a renda familiar

\begin{tabular}{lcc}
\hline Salários mínimos & $\mathrm{f}$ & $\%$ \\
\hline $1-3$ & 25 & 27,8 \\
$4-6$ & 25 & 27,8 \\
$7-9$ & 16 & 17,8 \\
$10-12$ & 13 & 14,4 \\
$13-15$ & 5 & 5,5 \\
$16-18$ & - & - \\
$19-20$ & 2 & 2,2 \\
$21-23$ & 1 & 1,1 \\
$>23$ & 2 & 2,2 \\
Não informado & 1 & 1,1 \\
\hline
\end{tabular}

Em relação à escolaridade (Tabela 5), observou-se que a ficha não refere, por exemplo, o nível de escolarização dos pais das crianças atendidas. Este fator pode influenciar muitos outros na relação terapeuta-cliente e não deveria ser negligenciado, na medida em que é esse responsável quem vai efetivamente envolver as crianças no atendimento.

Esta variação da escolaridade faz lembrar uma reflexão de Bezerra (1987), em relação à tendência equivocada do profissional de saúde mental a tomar a atividade psíquica como universal. Em suas palavras:

Para os profissionais de saúde que lidam no seu cotidiano com pessoas oriundas dos mais diversos estratos sociais, faixas etárias e regiões culturais, esta questão se torna fundamental. Nem sempre estaremos diante de pessoas com as mesmas noções que as nossas do adoecimento psíquico. (p. 141)

O mesmo autor destaca que, dependendo das expectativas e representações do paciente, pode-se trabalhar com maior ou menor facilidade no processo terapêutico. A rigor, o que Bezerra aponta é a necessidade de avaliações mais cuidadas a respeito da demanda do paciente e de sua origem social. E para nós esta avaliação deve incluir também o registro mais cuidadoso do nível de escolarização dos responsáveis pelas crianças e adolescentes que chegam ao NPA.

Os dados coletados revelam também que a maior parte dos pacientes possui formação incompleta do primeiro grau, conforme se observa na Tabela 6. Em segundo lugar, aparece o nível superior incompleto. Este último dado aponta para a grande procura efetivada pelos próprios alunos da UFES. A proximidade e a facilidade de acesso ao NPA talvez possa
Tabela 5

Distribuição de freqüência conforme a escolaridade

\begin{tabular}{|c|c|c|c|c|}
\hline \multirow{2}{*}{ Escolaridade } & \multicolumn{2}{|c|}{ Adultos } & \multicolumn{2}{|c|}{ Crianças } \\
\hline & $\mathrm{f}$ & $\%$ & $\mathrm{f}$ & $\%$ \\
\hline Terceiro grau & 6 & 6,7 & - & - \\
\hline $\begin{array}{l}\text { Terceiro grau } \\
\text { incompleto }\end{array}$ & 21 & 23,3 & - & - \\
\hline Segundo grau & 7 & 7,8 & - & - \\
\hline $\begin{array}{l}\text { Segundo grau } \\
\text { incompleto }\end{array}$ & 6 & 6,7 & 4 & 4,4 \\
\hline Primeiro grau & 1 & 1,1 & 1 & 1,1 \\
\hline $\begin{array}{l}\text { Primeiro grau } \\
\text { incompleto }\end{array}$ & 3 & 3,3 & 37 & 41,1 \\
\hline $\begin{array}{l}\text { Informação } \\
\text { inexistente }\end{array}$ & 3 & 3,3 & 1 & 1,1 \\
\hline Total & 47 & 52,1 & 43 & 47,8 \\
\hline
\end{tabular}

articular a variável escolaridade com a renda familiar, já que foram encontradas faixas de maior poder aquisitivo. Os alunos da UFES podem estar dando a essa clientela perfis de renda familiar, idade e escolarização mais altos; possuiriam os outros serviços de atendimento público da rede este tipo de cliente?

Além disso, pode-se aventar a hipótese de que, por tratarse de um serviço universitário, a população mais escolarizada considere também a qualidade desse serviço e, por isso, o procure com maior freqüência. Esta é uma hipótese a ser confrontada com possíveis estudos, até o momento inexistentes, sobre a caracterização da clientela dos serviços de assistência da rede pública no município de Vitória.

Quanto ao motivo da consulta (Tabela 7), observou-se que a maior parte das queixas incluíam o "nervosismo" como categoria para nomear o sofrimento que demanda assistência, tanto quando se toma o grupo de adultos, quanto com relação às crianças/adolescentes: os acompanhantes parecem nomear assim o mal-estar observado (causado) em (por) seus filhos. A queixa a respeito do nervoso, em geral, vinha associada a outras, como por exemplo os problemas familiares. ${ }^{2}$

Duarte (1986), estudando a "vida nervosa das classes trabalhadoras urbanas", aponta para a possibilidade de se considerar o nervoso como campo próprio de significação. Mais do que referência a problemas ou distúrbios, ressalta que existem diferenças, nos grupos pesquisados por ele no estado do Rio de Janeiro (Acari, Jurujuba e Meio da Serra), entre “estar nervoso" e "ser nervoso". A primeira locução referindo-se a uma forma mais leve ou passageira de perturbação; a segunda, já articulando-se com “doente dos nervos" e "temperamento nervoso", indicaria um estado mais 
Tabela 6

Distribuição de freqüência conforme a profissão

\begin{tabular}{lcccc}
\hline \multirow{2}{*}{ Profissão } & \multicolumn{2}{c}{ Adultos } & \multicolumn{2}{c}{ Responsáveis } \\
\cline { 2 - 5 } & $\mathrm{f}$ & $\%$ & $\mathrm{f}$ & $\%$ \\
\hline $\begin{array}{l}\text { Em formação } \\
\text { (estudante) }\end{array}$ & 10 & 11,1 & - & - \\
$\begin{array}{l}\text { Profissão nível } \\
\text { superior }\end{array}$ & 3 & 3,3 & 8 & 8,9 \\
$\begin{array}{l}\text { Profissão de } \\
\text { escritório e } \\
\text { especializados }\end{array}$ & 20 & 22,2 & 8 & 8,9 \\
$\begin{array}{l}\text { Prof. semi - } \\
\text { especializada }\end{array}$ & & & & \\
$\begin{array}{l}\text { Prof. não } \\
\text { especializada }\end{array}$ & 5 & 5,5 & 3 & 3.3 \\
$\begin{array}{l}\text { Prendas domésticas } \\
\text { Aposentado }\end{array}$ & 7 & 7,8 & 18 & 20 \\
\hline \begin{tabular}{l} 
Total \\
\hline
\end{tabular} & 1 & 1,1 & 6 & 6,7 \\
\hline
\end{tabular}

permanente, com motivação mais interiorizada. E ainda revela que essa forma de representação do nervoso não é característica das classes trabalhadoras, podendo aparecer nas classes médias e nos grupos mais medicalizados.

Há uma questão, específica para o campo desta pesquisa, que poderia ser articulada com esse material: existiria a possibilidade de, na recepção de um paciente que "está nervoso", trazendo o nervoso como queixa, produzir-se uma interiorização desse estado - no sentido de Duarte - em função do tipo de serviço oferecido? Ou seja, na dificuldade de se identificar estas representações, ou de se avaliar bem a demanda num primeiro contato, transformar, por um atravessamento do serviço, o "estar nervoso" em "ser nervoso"? Esta possibilidade deve ser considerada também à luz do que nos diz Silva (1992), quando refere-se ao predomínio da clínica, entendida como sinônimo de psicoterapia, nos Cursos de Psicologia. No âmbito do NPA, qualquer tentativa de avaliar esse tipo de atravessamento tornava-se inviável, em razão da ausência de dados sobre o percurso do paciente por entre os projetos de estágio. Por exemplo: quantas crianças recebidas são efetivamente atendidas? A que tipo de intervenção são submetidas? Estas são perguntas que, considerados os registros disponíveis na época deste estudo, ficariam sem resposta.

No grupo de pacientes adultos, a segunda queixa mais freqüente relacionou-se a problemas familiares e quanto às crianças/adolescentes, apareceram as dificuldades de aprendizagem.

Quanto à forma de encaminhamento (Tabela 8), os dados desta pesquisa revelaram que a maior parte dos clientes buscam espontaneamente o NPA (43,3\%). Considerou-se como busca espontânea quando na ficha constava essa referência, ou quando aparecia um parente ou amigo indicando o NPA ou o próprio tratamento psicológico. Em segundo lugar, aparecem os encaminhamentos formais de profissionais de saúde $(19,9 \%$ - somados os médicos, psicólogos e profissionais do HUCAM) ${ }^{3}$. Destaque-se também os encaminhamentos feitos por outras instituições (indicadas na Tabela 8 como “outros”); as citadas foram Conselho Tutelar dos Direitos da Criança e do Adolescente, Projeto Vida ${ }^{4}$ e Lar Fabiano de Cristo ${ }^{5}$.

Embora a escola não tivesse destaque na forma de encaminhamento $(8,8 \%)$, as dificuldades de aprendizagem (Tabela 7) foram apontadas para o grupo de crianças/ adolescentes como o segundo motivo do total de demandas por atendimento psicológico.

Tabela 7

Distribuição de freqüência conforme o motivo da consulta

\begin{tabular}{|c|c|c|}
\hline \multirow{3}{*}{ Motivo da consulta } & \multirow{3}{*}{$\frac{\text { Adultos }}{\mathrm{f}}$} & \multirow[t]{2}{*}{ Crianças } \\
\hline & & \\
\hline & & $\mathrm{f}$ \\
\hline Nervosismo & 11 & 13 \\
\hline Problemas de aprendizagem & - & 12 \\
\hline $\begin{array}{l}\text { Dificuldades de relacionamento } \\
\text { familiar }\end{array}$ & 10 & 6 \\
\hline Depressão & 9 & - \\
\hline $\begin{array}{l}\text { Dificuldades de relacionamento com o } \\
\text { parceiro }\end{array}$ & 2 & - \\
\hline $\begin{array}{l}\text { Dificuldades nos relacionamentos em } \\
\text { geral }\end{array}$ & 8 & 3 \\
\hline Sintomas psicossomáticos & 5 & 6 \\
\hline Problemas sexuais & 2 & - \\
\hline Uso de drogas & 2 & - \\
\hline Problemas financeiros & 2 & - \\
\hline Auto - conhecimento & 9 & - \\
\hline Medos & - & 1 \\
\hline Perda por morte/separação & - & 2 \\
\hline Vítima de violência & - & 1 \\
\hline Timidez & - & 6 \\
\hline
\end{tabular}


Tabela 8

Distribuição de freqüência conforme a origem do encaminhamento

\begin{tabular}{lcccc}
\hline \multirow{2}{*}{ Encaminhamento } & \multicolumn{2}{c}{ Adultos } & \multicolumn{2}{c}{ Crianças } \\
\cline { 2 - 5 } & $\mathrm{f}$ & $\%$ & $\mathrm{f}$ & $\%$ \\
\hline Procura espontânea & 30 & 33,3 & 9 & 10 \\
Médico & 1 & 1,1 & 10 & 11,1 \\
Escola & - & - & 8 & 8,8 \\
Aluno de Psicologia/UFES & 4 & 4,4 & 2 & 2,2 \\
& 3 & 3,3 & 3 & 3,3 \\
Funcionário da UFES & 2 & 2,2 & 2 & 2,2 \\
Profissionais HUCAM/UFES & 1 & 1,1 & 2 & 2,2 \\
Psicólogo & 1 & 1,1 & 1 & 1,1 \\
Sem informação & 5 & 5,6 & 6 & 6,7 \\
Outros & 47 & 52,1 & 43 & 47,8 \\
\hline Total & & & & \\
\hline
\end{tabular}

A constatação mais importante, porém, relaciona-se com o fato de que $43,3 \%$ da clientela busca o NPA espontaneamente. Se o Núcleo não tem vínculo formal com o SUS, isso se torna mais relevante e parece corroborar a idéia de que o NPA faz parte, ainda que informalmente, da rede e isto nos remete, necessariamente, à questão política. Estaria o NPA desvinculado das políticas de saúde mental implementadas na região em que está inserido? E se estas articulações existem, poderíamos tomar os programas de estágio curricular como condição ou consequiência delas?

\section{Considerações Finais}

O perfil da clientela levantado ao final desta pesquisa revela que o NPA/UFES recebeu, em 1996, pessoas que, em sua maioria, buscaram espontaneamente a instituição, estavam em idade economicamente ativa, apresentavam sofrimento psíquico caracterizado como "nervoso" e associado a dificuldades nas relações familiares (adultos) e a problemas de aprendizagem (crianças). Além disso, a clientela encontrava-se na faixa de renda familiar situada entre 0 e 6 salários mínimos, apresentando também curto tempo de escolarização. As profissões que predominaram para os pacientes adultos foram as especializadas/de escritório e para os responsáveis pelas crianças as profissões não especializadas.

Quanto à predominância de crianças e jovens na clientela atendida, percebe-se total sintonia com os dados encontrados tanto nos estudos anteriores realizados em clínicas-escola, quanto na rede de assistência mais ampla, como nos dizem Boarini e Borges (1998). Numa visão diferente da exposta por
Carvalho e Térzis (1989) - que ressaltaram em suas conclusões a possibilidade de crises psicológicas em momentos de transição -, Boarini e Borges assinalam a carência de estudos epidemiológicos relacionados ao adoecimento psíquico de crianças e adolescentes em nosso país. Isto as faz empreender uma interessante discussão a respeito do caráter histórico da infância e da crise hoje existente nesse domínio. Afirmam ainda, que, em nosso meio,

... Tal crise - denotada por violências (abusos, abandonos, agressões etc.) contra a criança em geral, além de extermínio, exploração pelo trabalho, fome, encaminhamentos equivocados de alunos que supostamente apresentam problemas na escola, preconceitos, estigmatização contra as crianças pertencentes às classes populares, em que estas, muitas vezes, passam a ser o "bode expiatório" do fracasso das instituições sociais - depõe contra qualquer possibilidade de garantia de direitos adquiridos ou reivindicação dos mesmos. (p. 103)

$\mathrm{Na}$ verdade, esse posicionamento traz, como desdobramento para as clínicas-escola, uma reflexão a respeito de como vem sendo atendida essa demanda. Além disso, aponta a necessidade de articulações dos serviços oferecidos com outras instituições/setores. Vale lembrar que em nossa amostra as crianças e adolescentes (0-19 anos) representaram $51,1 \%$ do total de atendimentos.

Um outro resultado interessante foi a completa ausência de idosos na clientela. Tal fato pode estar relacionado à falta de oportunidades para o idoso, em geral, na própria sociedade e não apenas em um serviço de atendimento em saúde prestado por um órgão da universidade. Resta, no entanto, investigar se o oferecimento de algum tipo de atenção para idosos poderia fazer aparecer essa parte da população e de que maneira essa demanda seria expressa.

Em desacordo com os estudos anteriores, encontrou-se um número quase equivalente de pacientes do sexo masculino e feminino. Esse resultado merece atenção, já que nas análises anteriores - e para explicar a recorrente ausência de homens nas amostras - era comum a referência a determinadas cobranças sociais sobre os homens, que tendiam a expressar menos seu mal-estar psíquico. Esse tipo de análise, para a amostra estudada nesta pesquisa, não nos parece útil. Talvez se possa levantar como hipótese, - numa aproximação à análise feita por Boarini e Borges (1998) com relação às crianças/adolescentes - a existência de uma crise na noção de masculinidade e/ou dificuldades na manutenção do antigo lugar social reservado aos homens (provedor, chefe da família, etc.). Em uma sociedade de classes como a nossa, valeria investigar de maneira mais acurada se tal quadro pode ser relacionado às intensas mudanças observadas no mundo do trabalho. As conseqüências dessas mudanças no volume e na qualidade da demanda de atenção em saúde mental, ainda resta pouco sistematizada. Tal hipótese também poderia explicar o grande número de universitários na clientela.

Com relação ao acesso aos dados, ressalte-se que, no momento da pesquisa, ainda não era possível desenvolver no NPA/UFES pesquisas que exigissem registros para além 
da ficha de triagem. Um estudo que objetivasse, por exemplo, identificar o índice de desistências ou engajamento nos tratamentos propostos estaria inviabilizada por absoluta falta de dados. Os registros existentes à época, portanto, eram passíveis de aperfeiçoamento no sentido da manutenção de prontuários individuais com dados sobre o percurso dos paciente nos diversos projetos de estágio existentes. Isto facilitaria o diálogo com outras instituições de saúde onde o paciente também fosse atendido. Uma outra função do registro mais cuidadoso seria a própria avaliação dos projetos de estágio e sua articulação com as demandas da comunidade. Concordamos com Pitta (1996) quando se refere aos processos de avaliação de dispositivos de atenção em saúde mental no Brasil como algo conceitualmente atravessado de imprecisões. No entanto, a autora ratifica a importância das avaliações como uma chance para produção de conhecimento para o campo.

A invenção permanente dos serviços em saúde mental, referida anteriormente (Saraceno et al., 1994), articula-se, a nosso ver, com a afirmação de Pitta (1996) a respeito da importância da avaliação em saúde mental. Se na área de saúde como um todo estas avaliações ainda não se tornaram rotina - vez que tal debate é relativamente recente no país -, mais ainda na área específica de saúde mental ${ }^{7}$, onde se necessita investir em etapas anteriores, aquelas mesmas às quais nos referíamos, de sistematização de dados.

A realidade do NPA não é, certamente, a de um serviço de saúde, mas entendemos que muito da experiência que acontece em ambulatórios e postos de saúde pode ser aproveitada no treinamento de universitários, e vice-versa. A vivência do aluno-estagiário num Núcleo/Serviço de Psicologia Aplicada é, em geral, a primeira referência formal da prática profissional futura. E tanto mais produtiva será, quanto mais sintonizada com as novas demandas técnicas e políticas do campo da saúde mental.

\section{Referências}

Bezerra Jr., B. (1987). Considerações sobre terapêuticas ambulatoriais em saúde mental. In S. Tundis \& N. Costa (Orgs.), Cidadania e loucura. Políticas de saúde mental no Brasil (pp. 133-169). Petrópolis: Vozes/ ABRASCO.

Boarini, M. L., \& Borges, R. F. (1998). Demanda infantil por serviços de saúde mental: sinal de crise. Estudos de Psicologia (Natal), 3(1), 83-108.

Brasil, M. A. A. (1998). Programa de Residência em Hospital Geral. Cadernos IPUB (Rio de Janeiro: Instituto de Psiquiatria/UFRJ), número especial, 71-77.

Carvalho, R. M. L. L. \& Térzis, A. (1989). Caracterização da clientela atendida na Clínica Psicológica do Instituto de Psicologia da PUCCAMP - II. Estudos de Psicologia (Campinas), 6(1), 95-110.

Duarte, L. F. D. (1986). Da vida nervosa nas classes trabalhadoras urbanas. Rio de Janeiro: Jorge Zahar.

Fagundes, H., \& Libério, M. A. (1997). A reestruturação da assistência na cidade do Rio de Janeiro. Estratégias de construção e desconstrução. Saúde em foco - Informe Epidemiológico em Saúde Coletiva (Rio de Janeiro: Secretaria Municipal de Saúde), 16, 30-35.

Lopez, M. A. (1983). Características da clientela de clínicas-escola de Psicologia em São Paulo. Arquivos Brasileiros de Psicologia, 35(1), 78-92.

Pitta, A. M. F. (1996). Cuidados com o cuidar: estratégias de avaliação. Cadernos IPUB (Rio de Janeiro: Instituto de Psiquiatria/UFRJ), 3, 185195.

Cadernos IPUB (Rio de Janeiro: Instituto de Psiquiatria/UFRJ), 1998, número especial.

Santos, M. A (1990). Caracterização da clientela de uma clínica psicológica da Prefeitura de São Paulo. Arquivos Brasileiros de Psicologia, 42 (2), 79-94

Santos, M. A., Moura, L., Pasian, S. R., \& Ribeiro, P. L. L. (1993). Caracterização da clientela de adolescentes e adultos de uma clínica-escola de Psicologia. Psicologia: Teoria e Pesquisa, 9(1), 123-144.

Saraceno, B., Asioli, F., \& Gianni, T. (1994). Manual de saúde mental. São Paulo: Hucitec.

Silva, R. C. (1992). A formação em psicologia para o trabalho em Saúde Pública. In F. Campos (Org.), Psicologia e saúde. Repensando práticas (pp.25-40). São Paulo: Hucitec.

Urtiaga, M. E., Almeida, G., Vianna, M. E. D., Santos, M. V., \& Botelho, S. (1997). Fatores preditivos de abandono em psicoterapias. Um estudo na Clínica Sérgio Abuchaim. Jornal Brasileiro de Psiquiatria, 6(5), 279-283.

Notas

1. O salário-mínimo à época correspondia a cerca de cem reais.

2. Quando isso ocorria, computava-se cada uma das queixas nos itens próprios.

3. Este é um grande hospital universitário, vinculado à UFES.

4. Projeto de atenção a comunidades carentes, vinculado à Igreja Católica.

5. Projeto de assistência à população de baixa renda, organização não estatal.

6. Ressalvem-se algumas poucas iniciativas neste sentido em nosso meio como a do LAPSO/IPUB/UFRJ.

Rita de Cássia Ramos Louzada, mestre em Psicologia Social pelo Instituto de Psicologia da Universidade Federal do Rio de Janeiro e doutoranda do Programa de Pós-graduação em Psiquiatria e Saúde Mental - IPUB/ UFRJ, é professora no Departamento de Psicologia Social e do Desenvolvimento da Universidade Federal do Espírito Santo. Endereço para correspondência: Rua Cláudio da Costa, 150; Rio de Janeiro, RJ; CEP 21235450. Telefax: (21) 3371-9187.E-mail: ritacrl@uol.com.br 